\title{
Gait Analysis after Total Knee Arthroplasty with Posterior Cruciate Ligament Retention Rotating Platform and Fixed Total Knee Arthroplasty
}

\author{
GUO. QIANG. LIU, HAO. PAN*, Z. LIU1, J. J. WANG, F. WANG, P. WANG, QIU LING SHI, JIN GUO AND LIN LI \\ Cangzhou Hospital of Integrated TCM-WM, Cangzhou, Hebei 061001 China, 1Lulong County Hospital,Lulong, Hebei 066400, \\ China
}

Liu et al.: Gait analysis after total knee arthroplasty

\begin{abstract}
To investigate the gait analysis of patients after total knee arthroplasty with posterior cruciate ligament retention rotating platform and fixed total knee arthroplasty, so as to provide ideas for clinical rationalization treatment. A total of 82 patients with posterior cruciate ligament preserving rotating and fixed platforms were selected for knee replacement in our hospital and were followed up for 1 y. The knee joint Hospital for special surgery score, Western Ontario and McMaster Universities Osteoarthritis index score and the application of a three-dimensional motion capture system were used to compare the parameters of knee joint kinematics and dynamics. In the application of knee arthroplasty between the posterior cross retained rotating platform and the fixed platform, the temporal and spatial parameters and motion parameters in the knee function and gait analysis were significantly improved compared with those before the operation and the differences were statistically significant $(p<0.05)$, while there was no statistically significant difference between the two groups $(p>0.05)$. Both rotating platform and fixed platform can significantly improve the knee joint function and gait of patients with knee arthroplasty using posterior cruciate ligament retention prosthesis.
\end{abstract}

Key words: Rotating platform, fixed platform, knee replacement, gait analysis

At present, total knee arthroplasty as a routine treatment of end-stage knee osteoarthritis has achieved good therapeutic effect. According to different principles, the design of knee prosthesis can be divided into two types: posterior cruciate ligament replacement prosthesis (PS) and posterior cruciate ligament retention prosthesis (CR). According to the activity of the spacer, it can be divided into rotating platform prosthesis (RPP) and fixed bearing prosthesis (FBP). In the past, PS prosthesis has been widely used in clinical practice and then the research on rotating platform and fixed bearing is also more in depth, while the research on rotary platform and fixed bearing of CR prosthesis is relatively less. There are few reports on the kinematics and dynamics of patients after knee joint replacement with CR prosthesis rotating platform and fixed platform using Three-Dimensional (3D) motion capture system. In this study, a follow up observation was conducted on the gait analysis after posterior cruciate ligament reservation rotating platform and fixed total knee arthroplasty and the following reports are presented. A total of 82 patients with posterior cruciate ligament- sparing knee arthroplasty in our hospital from June 2018 to June 2019 were selected and randomly divided into 42 patients in group A (Rotating platform group), including 12 males and 30 females, with an average age of 63.4 \pm 3.6 y. Group B (Fixed platform group) included 42 patients, including 11 males and 31 females, with an average age of $62.1 \pm 4.6 \mathrm{y}$. There was no significant difference in general data between the two groups. Patients in Group A received knee arthroplasty with link anatomical rotary platform knee prosthesis (GEMINI MK II), while patients in Group B were treated with Triathlon prosthesis of STRYKER Company. All the patients were operated by a senior chief surgeon. The 3D gait of all patients was analyzed by using Foot plantar pressure analysis system (Belgium), Advanced Mechanical Technology Inc (AMTI) 3D lateral stand (USA), Charnwood Dynamics Ltd active 3D infrared motion capture system (USA) and Delsys lnc.16 channel wireless surface electromyography (USA) (Table 1). The Hospital for special surgery (HSS) score, Western Ontario and McMaster Universities Osteoarthritis index (WOMAC) were recorded before 
and 1 y after operation. Gait analysis indicators include spatiotemporal parameters (gait speed, stride frequency, step length, gait cycle, single support time, double support time) and kinematic parameters (knee joint motion angle).SPSS 22.0 statistical software was used for analysis. Paired t test was used for intra group comparison and independent sample t test for inter group comparison. Count data $(\mathrm{x} \pm \mathrm{s})$ showed that the difference was statistically significant $(\mathrm{p}<0.05)$. For comparison, the peak value is taken as absolute value. All patients were successfully followed up and there were statistically significant differences between the two groups in HSS score and WOMAC score before and 1 y after operation $(\mathrm{p}<0.05)$. Postoperative comparison between the two groups showed no statistical difference $(\mathrm{p}>0.05)$, as shown in Table 2 . The walking speed, stride frequency, step length, gait cycle, single support time and double support time of the two groups were significantly improved compared with those before operation $(p<0.05)$. There was no significant difference in the reference indexes between groups ( $p>0.05$ ), as shown in Table 3. The postoperative kinematic parameters of knee joint movement angle of the two groups were higher than those before operation, with statistical difference $(\mathrm{p}<0.05)$. There was no significant difference between the two groups ( $p>0.05)$, as shown in Table 4. With the development of aging, knee osteoarthritis seriously affects the quality of daily life of patients. Knee arthroplasty as the end treatment of knee osteoarthritis patients, the curative effect is significant ${ }^{[1,2]}$. Among the knee prosthesis, the posterior cruciate ligament retention prosthesis (CR) has been favored by more and more orthopedics doctors for its advantages of retaining more bone mass and maintaining proprioception of patients. CR prosthesis can be divided into rotating platform type and fixed bearing type. Some studies have shown that the fixed platform tibial prosthesis is not a high polished surface, because the locking mechanism between the pad and the tibial prosthesis cannot simulate the motion characteristics of the knee joint during flexion and extension, which is easy to cause pad wear and increase the risk of surgical failure ${ }^{[3,4]}$. However, the rotating platform prosthesis, accompanied by certain rotation in the process of knee joint flexion and extension, causes stress dispersion, greatly reduces the wear of polyethylene liner and has significant curative effect. Despite the theoretical advantages of knee arthroplasty with rotating platform prosthesis, Zhang Chengzhang ${ }^{[5]}$ believed that both RP and FB applied to Total Knee Arthroplasty (TKA) for the treatment of end-stage knee Hyaluronate Injections, Platelet-Rich Plasma (PRP) Injections, Mesenchymal Stem Cells, or MSCs and autologous Cultured Chondrocytes are few advanced options availabIe for the treatment of advanced osteoarthritis could significantly improve knee joint function, significantly improve or

TABLE 1: COMPARISON OF GENERAL DATA BETWEEN THE TWO GROUPS

\begin{tabular}{lccccc}
\hline Groups & Cases & Gender & Age & Weight & Height \\
\hline & & (Female/Male) & (y old) & (kg) & (cm) \\
\hline Group A & 42 & $30 / 12$ & $63.4 \pm 3.6$ & $60.1 \pm 4.5$ & $162.2 \pm 4.5$ \\
Group B & 42 & $31 / 11$ & $62.1 \pm 4.6$ & $63.2 \pm 3.6$ & $160.4 \pm 5.6$ \\
$\mathrm{t}$ & & 1.342 & 2.080 & 0.307 & 2.122 \\
$\mathrm{p}$ & & 0.672 & 0.052 & 0.673 & 0.061 \\
\hline
\end{tabular}

TABLE 2: COMPARISON OF HSS SCORES AND WOMAC SCORES BETWEEN THE TWO GROUPS BEFORE AND ONE YEAR AFTER OPERATION

\begin{tabular}{lccccc}
\hline \multirow{2}{*}{ Groups } & \multirow{2}{*}{ Cases } & \multicolumn{2}{c}{ HSS scores } & \multicolumn{2}{c}{ WOMAC scores } \\
\cline { 3 - 6 } Group A & & Pre-operation & One year after operation & Pre-operation & One year after operation \\
Group B & 42 & $58.11 \pm 10.61$ & $82.14 \pm 7.60$ & $63.14 \pm 7.60$ & $22.21 \pm 3.45$ \\
\hline
\end{tabular}

TABLE 3: COMPARISON OF SPATIOTEMPORAL PARAMETERS BETWEEN THE TWO GROUPS

\begin{tabular}{lcccc}
\hline \multirow{2}{*}{ Spatiotemporal parameters } & \multicolumn{2}{c}{ Group A (42 cases) } & \multicolumn{2}{c}{ Group B (42 cases) } \\
\cline { 2 - 5 } Walking speed (m/s) & Pre-operation & One year after operation & Pre-operation & One year after operation \\
Step frequency (step/min) & $0.51 \pm 0.03$ & $0.83 \pm 0.02$ & $0.53 \pm 0.03$ & $0.82 \pm 0.03$ \\
Step length (m) & $80.21 \pm 13.12$ & $103.21 \pm 11.12$ & $81.23 \pm 13.24$ & $100.05 \pm 12.11$ \\
Gait cycle (s) & $0.52 \pm 0.12$ & $0.62 \pm 0.11$ & $0.51 \pm 0.09$ & $0.61 \pm 0.12$ \\
Single support time (s) & $1.21 \pm 0.16$ & $1.14 \pm 0.13$ & $1.22 \pm 0.12$ & $1.11 \pm 0.14$ \\
Double support time (s) & $0.37 \pm 0.08$ & $0.48 \pm 0.07$ & $0.38 \pm 0.06$ & $0.49 \pm 0.09$ \\
\hline
\end{tabular}


TABLE 4: COMPARISON OF PEAK AND MAXIMUM RANGE OF MOTION OF KNEE JOINT BETWEEN TWO GROUPS

\begin{tabular}{|c|c|c|c|c|c|}
\hline \multirow{2}{*}{$\begin{array}{l}\text { Three } \\
\text { dimensional } \\
\text { plane }\end{array}$} & \multirow[b]{2}{*}{ Movement angle } & \multicolumn{2}{|c|}{ Group A (42 cases) } & \multicolumn{2}{|c|}{ Group B (42 cases) } \\
\hline & & $\begin{array}{c}\text { Pre- } \\
\text { operation }\end{array}$ & $\begin{array}{c}\text { One year after } \\
\text { operation }\end{array}$ & Pre-operation & $\begin{array}{c}\text { One year after } \\
\text { operation }\end{array}$ \\
\hline \multirow{4}{*}{ Sagittal plane } & Initial angle & $10.54^{\circ} \pm 2.12^{\circ}$ & $5.48^{\circ} \pm 1.55^{\circ}$ & $9.46^{\circ} \pm 2.31^{\circ}$ & $6.11^{\circ} \pm 1.12^{\circ}$ \\
\hline & Peak value of buckling angle & $78.11^{\circ} \pm 4.56^{\circ}$ & $95.54^{\circ} \pm 2.33^{\circ}$ & $76.32^{\circ} \pm 4.12^{\circ}$ & $96.32^{\circ} \pm 3.42^{\circ}$ \\
\hline & Peak value of stretching angle & $6.95^{\circ} \pm 2.12^{\circ}$ & $1.54^{\circ} \pm 0.98^{\circ}$ & $6.44^{\circ} \pm 2.32^{\circ}$ & $1.74^{\circ} \pm 1.12^{\circ}$ \\
\hline & Maximum range of activity & $75.11^{\circ} \pm 4.54^{\circ}$ & $90.44^{\circ} \pm 2.12^{\circ}$ & $76.23^{\circ} \pm 3.55^{\circ}$ & $91.15^{\circ} \pm 2.33^{\circ}$ \\
\hline \multirow{4}{*}{ Coronal plane } & Initial angle & $6.54^{\circ} \pm 1.12^{\circ}$ & $2.45^{\circ} \pm 1.33^{\circ}$ & $6.12^{\circ} \pm 1.23^{\circ}$ & $2.11^{\circ} \pm 1.12^{\circ}$ \\
\hline & Peak varus angle & $10.11^{\circ} \pm 2.43^{\circ}$ & $20.54^{\circ} \pm 2.64^{\circ}$ & $9.88^{\circ} \pm 2.62^{\circ}$ & $19.99^{\circ} \pm 2.86^{\circ}$ \\
\hline & Peak valgus angle & $1.75^{\circ} \pm 0.88^{\circ}$ & $2.86^{\circ} \pm 0.12^{\circ}$ & $1.54^{\circ} \pm 0.75^{\circ}$ & $2.73^{\circ} \pm 0.21^{\circ}$ \\
\hline & Maximum range of activity & $12.11^{\circ} \pm 4.02^{\circ}$ & $21.54^{\circ} \pm 2.44^{\circ}$ & $11.25^{\circ} \pm 3.12^{\circ}$ & $19.48^{\circ} \pm 3.12^{\circ}$ \\
\hline \multirow{4}{*}{ Horizontal plane } & Initial angle & $12.11^{\circ} \pm 5.43^{\circ}$ & $11.86^{\circ} \pm 3.12^{\circ}$ & $12.54^{\circ} \pm 4.63^{\circ}$ & $11.67^{\circ} \pm 3.55^{\circ}$ \\
\hline & Peak value of internal rotation angle & $4.43^{\circ} \pm 3.45^{\circ}$ & $21.88^{\circ} \pm 3.12^{\circ}$ & $5.54^{\circ} \pm 3.12^{\circ}$ & $21.74^{\circ} \pm 3.32^{\circ}$ \\
\hline & Peak value of external rotation angle & $15.34^{\circ} \pm 4.11^{\circ}$ & $18.54^{\circ} \pm 3.35^{\circ}$ & $14.77^{\circ} \pm 3.96^{\circ}$ & $19.43^{\circ} \pm 3.12^{\circ}$ \\
\hline & Maximum range of activity & $19.67^{\circ} \pm 3.25^{\circ}$ & $40.11^{\circ} \pm 4.50^{\circ}$ & $19.32^{\circ} \pm 4.12^{\circ}$ & $41.42^{\circ} \pm 5.23^{\circ}$ \\
\hline
\end{tabular}

disappear the postoperative knee joint pain and improve the deformity such as knee pronation, with significant curative effect and high safety. In clinical work, the judgment of knee joint function is often based on the doctor's visual inspection or physical examination and patients' complaints. Patients' complaint of knee joint pain is a subjective feeling, originated from multilevel nervous system and is easily affected by cognitive and situational factors, which makes the clinical symptoms of knee osteoarthritis often inconsistent with the extent of anatomical structure damage ${ }^{[6]}$. The use of a $3 \mathrm{D}$ motion capture system to perform 3D gait analysis on patients with knee osteoarthritis helps to better understand knee kinematics and dynamics and other indicators, thereby providing important reference basis for the diagnosis, treatment and research of knee osteoarthritis ${ }^{[7]}$. The functional evaluation of patients undergoing knee arthroplasty often lacks quantitative analysis and 3D gait analysis can observe and analyze the gait, joint kinematics and dynamics of the human body when walking and provide a series of time, geometric, mechanical and other parameter values and curves, so as to objectively and quantitatively evaluate the human walking function ${ }^{[8-12]}$. In this study, by comparing the HSS score and WOMAC score of the two groups of patients, the knee joint function of the two groups was significantly improved compared with that before the operation. As for the comparison between groups, although the rotary platform prosthesis has theoretical advantages as mentioned above, there is no significant difference between the two groups from the results of this study, which may be related to the short follow up time of this study. In the gait analysis and comparison of the two groups, the spatiotemporal parameters and motion parameters were improved compared with those before operation, the difference was statistically significant, but there was no statistical difference between the two groups. In conclusion, for the posterior cruciate ligament retention prosthesis, whether it is a rotating platform or a fixed platform, the postoperative function and gait of patients can be greatly improved. The deficiency of this study lies in the lack of large sample multicenter study and short follow up time. The long-term efficacy of patients' needs further study.

\section{Acknowledgements:}

This work was supported by the Youth Science and Technology Project of Hebei Provincial Health and Family Planning Commission.

\section{Conflict of interests:}

The authors declared no conflict of interest.

\section{REFERENCES}

1. Papagiannis GI, Roumpelakis IM, Triantafyllou AI, Makris IN, Babis GC. No differences identified in transverse plane biomechanics between medial pivot and rotating platform total knee implant designs. J Arthroplasty 2016;31:1814-20.

2. Chen YZ. Mid and long-term follow-up of anatomic rotating platform knee prosthesis. Orthop J Chin 2016;24:1617-20.

3. Okamoto Y, Nakajima M, Jotoku T, Otsuki S, Neo M. Capsular release around the intercondylar notch increases the extension gap in posterior-stabilized rotating-platform total knee arthroplasty. Knee 2016;23:730-5.

4. Cheng JS, Li JX, Zhou YG, Wang HB, Pin LV. Clinical Effects 
of Prosthesis of Rotating Platform and Posterior Fixed Bearing on Patients Undergoing Total Knee Arthroplasty. Progress in Modern Biomedicine 2016;16:748-50.

5. Zhang CC, Liu Y, Wang F. Comparison of fixed and rotating platform prosthesis replacement in the treatment of knee osteoarthritis. Orthop J Chin 2018;11:1005-9.

6. Duan DS, Chen KF, Guo XD. Study on three-dimensional and kinematic characteristics of knee joint after proximal fibula osteotomy. Chin J Geriatr Orthop Rehabil 2017;3:162-6.

7. Huang $\mathrm{P}$, Wang $\mathrm{Y}$, Chen B. Three-dimensional motion analysis of patients with knee osteoarthritis. Chin Gen Pract 2020;23:2169-76.

8. Liu ZH, Zhang J, He C. Comparative analysis of gait after movable platform total knee prosthesis and unicondylar knee prosthesis replacement. Chin J Joint Surg 2017;11:17-23.

9. Guo YL. Three-position gait analysis of clinical efficacy of abdominal transcranial magnetic therapy for Parkinson's. J Med Theory Pract 2017;30:298-9.

10. Shi HJ, Huang HS, Yu YY. Gait analysis of patients with simple anterior cruciate ligament rupture and meniscus injury. Chin J Sport Med 2017;36:858-64.

11. Du LL, Xia Q. Kinematic characteristics analysis of knee hyperextension gait in stroke patients with hemiplegia. Chinese J Rehabil 2018;33:7-10.

12. Li Y, Fang XH, Mao RL. Study on the difference of threedimensional gait parameters between elderly patients with normal pressure hydrocephalus and patients with stroke. Geriatr Health Care 2017;23:324-7.

This is an open access article distributed under the terms of the Creative Commons Attribution-NonCommercial-ShareAlike 3.0 License, which allows others to remix, tweak, and build upon the work non-commercially, as long as the author is credited and the new creations are licensed under the identical terms

This article was originally published in a special issue, "Clinical Research in Pharmaceutical and Biomedical Sciences" Indian J Pharm Sci 2021:83(1)Spl issue1;58-61 